\title{
Combining earthquake forecasts using differential probability gains
}

\author{
Peter N Shebalin ${ }^{1,2^{*}}$, Clément Narteau ${ }^{2}$, Jeremy Douglas Zechar ${ }^{3}$ and Matthias Holschneider ${ }^{4}$
}

\begin{abstract}
We describe an iterative method to combine seismicity forecasts. With this method, we produce the next generation of a starting forecast by incorporating predictive skill from one or more input forecasts. For a single iteration, we use the differential probability gain of an input forecast relative to the starting forecast. At each point in space and time, the rate in the next-generation forecast is the product of the starting rate and the local differential probability gain. The main advantage of this method is that it can produce high forecast rates using all types of numerical forecast models, even those that are not rate-based. Naturally, a limitation of this method is that the input forecast must have some information not already contained in the starting forecast. We illustrate this method using the Every Earthquake a Precursor According to Scale (EEPAS) and Early Aftershocks Statistics (EAST) models, which are currently being evaluated at the US testing center of the Collaboratory for the Study of Earthquake Predictability. During a testing period from July 2009 to December 2011 (with 19 target earthquakes), the combined model we produce has better predictive performance - in terms of Molchan diagrams and likelihood - than the starting model (EEPAS) and the input model (EAST). Many of the target earthquakes occur in regions where the combined model has high forecast rates. Most importantly, the rates in these regions are substantially higher than if we had simply averaged the models.
\end{abstract}

Keywords: Probabilistic forecasting; Earthquake interaction; Forecasting and prediction; Statistical seismology

\section{Background}

Despite a growing number of reasonably reliable and skillful numerical seismicity forecast models, operational earthquake forecasting remains a daunting challenge. One of the fundamental difficulties is that operational forecasts require high expected earthquake rates to make substantial decisions (e.g., evacuation or other emergency actions), but the probabilities derived from statistical seismicity models are still quite small (Jordan and Jones 2010). One potential approach to this problem is to combine models in a way that maximizes overall predictive skill.

It is well known that combining many models or classifiers that describe the same data may yield higher performances than any individual member. These ensemble learning techniques include methods such as Bayesian

\footnotetext{
${ }^{*}$ Correspondence: shebalin@mitp.ru

${ }^{1}$ Institute of Earthquake Prediction Theory and Mathematical Geophysics, 84/32 Profsouznaya, Moscow 117997 Russia

2 Equipe de Dynamique des Fluides Géologiques, Institut de Physique du Globe de Paris, Sorbonne Paris Cité, Univ Paris Diderot, UMR 7154 CNRS, 1 rue Jussieu, Paris 75238, France

Full list of author information is available at the end of the article
}

model averaging (Marzocchi et al. 2012) and, in the context of classifiers, boosting (Hastie et al. 2008). In the first case, the combined model is a weighted sum of individual posterior probabilities, the weights being new parameters that can be learned from the data. For boosting, weighted data are used to train a collection of classifiers; during iteration, previously misclassified data get higher weight. These approaches are similar in that the combined models or classifiers are of the same abstract nature, meaning that each gives a statistical description of the same data set. In our approach, we combine models of different natures. For example, alarm-based and rate-based forecast models both give a description of the seismicity in a region. However, alarm-based models do this through a space-time alarm function, generally not normalized, whereas rate-based models give a description in terms of space-time Poisson event rates. The combination of two such models cannot be cast into the classical form used in ensemble techniques. Furthermore, the vast majority of current seismicity models are based only on catalogs of past earthquakes, and there is some hope that additional geological, geodetic, and physical information could

\section{量 Springer}

(c) 2014 Shebalin et al: licensee Springer. This is an Open Access article distributed under the terms of the Creative Commons Attribution License (http://creativecommons.org/licenses/by/2.0), which permits unrestricted use, distribution, and reproduction in any medium, provided the original work is properly credited. 
improve forecast performance. Combining such information should be done in a complementary fashion so as not to increase uncertainty and thereby degrade forecast performance. This challenge cannot be met by traditional methods, and a new method to combine forecasts should identify what additional information a model can contribute to an existing forecast.

It is often difficult to verify the presence of additional predictive information in an earthquake forecast model, but researchers are attempting to address this problem with the Collaboratory for the Study of Earthquake Predictability (CSEP). In CSEP testing regions around the world (e.g., California, Italy, New Zealand, the western Pacific, Japan, and the globe), various forecast models are evaluated in a standardized way (Jordan 2006; Gerstenberger et al. 2007; Zechar et al. 2010a; Zechar and Jordan 2010; Zechar et al. 2010b; Rhoades and Gerstenberger 2009; Nanjo et al. 2011; Tsuruoka et al. 2012; Eberhard et al. 2012; Taroni et al. 2014). One subtle benefit of these centers is that all forecasts are systematically archived. Therefore, one can test methods of combination using archived prospective forecasts. Most forecasts within CSEP testing centers are rate-based forecast models with a time step of 1 day, 3 months, or 5 years, and testing regions are gridded with square cells of $0.1^{\circ}$ and a class interval of earthquake magnitude of 0.1 from $M \geq 3.95$ earthquakes. For a much longer period, several alarm-based models have been developed and tested by various research groups. Known examples are the global and regional tests of $\mathrm{M} 8, \mathrm{CN}$, and RTP (Keilis-Borok and Kossobokov 1990; Keilis-Borok and Rotwain 1990; Peresan et al. 1999; Shebalin et al. 2006; Romashkova and Kossobokov 2004; Zechar 2010).

Researchers have suggested a few methods for combining models and/or earthquake precursors. For a set of rate-based models, a weighted average is a natural solution (Rhoades and Gerstenberger 2009; Marzocchi et al. 2012; Rhoades 2013). In the current implementation of such approaches weights do not depend on space; rather, they are chosen according to a relative performance of the model observed during some testing period. A direct product of functions describing precursory behavior was used in the RTL prediction algorithm (Sobolev et al. 1996). In this case, even if the initial functions are probabilistic, the output is a nonprobabilistic alarm-based model. Another way to combine models is by using Bayes' formula for conditional probabilities (Sobolev et al. 1991). However, when using this approach, it is difficult to take into account the interdependence of the combined elements, and resulting estimates are hardly probabilistic.

Shebalin et al. (2011) suggested a method based on differential probability gains to convert alarm-based to rate-based earthquake forecast models. Thus, nonprobabilistic forecast models or seismic precursor maps can be converted to probabilistic rate-based models. In this article, we generalize this differential probability gains approach to combine all types of time-varying forecasts.

\section{Methods}

\section{Evaluation of forecast models}

Following the CSEP standards, we discretized forecasts in space and time according to a predefined grid and a given time step. In prospective tests, all forecasts are given for the next time step and a finite magnitude range.

\section{Molchan tests}

Molchan tests are used to compare an alarm-based model with a reference model of seismicity defined on the same spatial grid (Molchan 1990). For any space-time region $(x, t)$, the reference model provides the rate $\lambda(x, t)$ of target earthquakes. The alarm-based model is entirely defined by its alarm function $A(x, t)$. Where this alarm function exceeds a given threshold value $A_{0}$, an alarm is issued and a target earthquake is expected to occur. Although it is not necessary, it is usually assumed that $A$ values are ordered from smallest to largest according to the probability of occurrence of a target event. In almost all cases, numerical forecast models can be easily converted to an alarm-based forecast because the information provided by a numerical value assignment on a given space-time grid can be used as an alarm function.

A Molchan test takes the form of a diagram comparing rates of types I and II errors for varying threshold values $A_{0}$ (i.e., a level of alarm). For different $A_{0}$ values, type I errors are measured by

$$
\tau\left(A_{0}\right)=\frac{\sum_{A(x, t) \geq A_{0}} \lambda(x, t)}{\sum \lambda(x, t)},
$$

where the sum symbols refer to the space-time regions in which the subscript condition is satisfied. The $\tau$ value is often interpreted as a fraction of the space-time region occupied by alarms (Kossobokov and Shebalin 2003; Molchan 2010). Here, it is important to emphasize that this fraction is given by a reference model that may depend on time. Type II error rates are the miss rates $v\left(A_{0}\right)$, the fraction of target earthquakes that occurred in space-time bins in which $A<A_{0}$.

In a Molchan diagram, the $(\tau, v)$ curve constructed for all $A_{0}$ values is called the Molchan trajectory (Molchan 1990; Zechar and Jordan 2008). This trajectory runs from the point $(0,1)$ to the point $(1,0)$ for a decreasing $A_{0}$ value. The diagonal connecting these two points corresponds to an unskilled forecast. Below this diagonal, the alarm function may bring additional predictive power (Shebalin et al. 2006) with respect to a given level of significance $\alpha$ (Zechar and Jordan 2008, Equations 2 and 3). 
The closer the Molchan trajectory is to the $y$ axis, the more skill the forecast has. It is often desirable to characterize forecast skills by a scalar value, the so-called loss function. Examples of loss functions are the minimal summary error (Molchan 1990, $\max (1-\tau-v)$ ), the minimax loss function (Molchan 1990, $\inf (\max (\nu, \tau))$ ), the area above the Molchan curve (Zechar and Jordan 2008; 2010), and the maximal probability gain (Aki 1996, $\max ((1-v) / \tau))$. If there are a large number of target events, we can also suggest here the target-weighted probability gain (i.e., $\left.\max \left((1-v)^{2} / \tau\right)\right)$. Note the singularity at $\tau=0$ for many of these expressions. Furthermore, there is always a trade-off between the rates of false alarms and failures to predict so that the best scalar value may depend on the goal of the forecast (Molchan 1990). In all cases, one should plot the two-dimensional Molchan diagram to visualize the vector data that are processed.

\section{Likelihood tests}

Likelihood tests are commonly used to evaluate ratebased models of seismicity. Their forecasts are tested against the number $\omega(x, t)$ of observed target earthquakes in each bin (Schorlemmer et al. 2007). For simplicity, individual rates are assumed to follow independent Poisson processes. For all magnitude classes, the complete likelihood counts the Poisson joint log-likelihood of the observed number $\omega(x, t)$ given the forecast $\lambda(x, t)$ :

$$
L(t)=\sum(-\lambda(x, t)+\omega(x, t) \log (\lambda(x, t))-\log (\omega(x, t) !)
$$

The closer the joint log-likelihood is to zero, the better the forecast is.

The spatial likelihood is a reduction of the complete likelihood applied to a forecast with rate value normalized to match the total observed number of targets (Zechar et al. 2010a). In each spatial bin, the single rate values are obtained by summing the expected event rates over the whole range of magnitudes (Kossobokov 2006). For the total duration of the experiment, the total sum of log likelihoods over all time steps can be calculated and divided by the total number of observed events to estimate a log likelihood per earthquake.

Likelihood tests applied to forecasts defined on a highdensity spatial grid are often criticized because of potential earthquake interactions (Molchan 2012). However, the problem of bin independence cannot be solved easily and it is generally thought that the dependence is conditional on earthquake occurrence. For example, we expect many aftershocks after large earthquakes, but a prospective forecast experiment requires any interbin dependence to be provided in advance, before one knows about the large earthquake (Zechar 2010).

\section{Testing forecast models at CSEP}

Likelihood and Molchan tests are complementary and both can be used to estimate the performance of the forecast models. The likelihood tests, however, do not apply to nonprobabilistic alarm-based models.

At CSEP testing centers, all the rate-based models are evaluated by using likelihood tests. In contrast, alarmbased models are only tested at the California testing center using Molchan tests and the related ROC and ASS tests (Zechar and Jordan 2008; Zechar 2010). Unfortunately, alarm- and rate-based models are still tested independently. The main reason for this is that the likelihood tests cannot be applied to an alarm-based model with a nonprobabilistic alarm function. To address this problem, Shebalin et al. (2012) proposed a method to convert alarm-based models to rate-based forecasts. In addition, two rate-based models can be compared by using Molchan diagrams. In practice, the complete ratebased model should be reduced to a single rate value by summing over a given range of magnitude. The reduced model can be treated as a rate-based model and/or as an alarm-based model. Its alarm function is simply composed of the single rate value in each spatial cell.

In summary, all requirements are satisfied for systematic implementation of both likelihood and Molchan tests. The next challenge is to identify the regions of particular skill for each model and combine models in a way that increases the expected event rates.

\section{A differential probability gain approach for combining two earthquake forecast models}

Here, we generalize the concept of differential probability gain to combine different types of earthquake forecast models. The main idea is to successively create new generations of a rate-based model by injecting into the current generation the additional information provided by other input models. In what follows, we describe one iteration of the combination procedure using a current model (the initial rate-based forecast model at the first iteration) and an input model (any type of numerical forecast model) to compute a new rate-based forecast model.

The entire procedure is based on the Molchan diagram that evaluates retrospectively the performance of the input model with respect to the current model. For this reason, the input model must be expressed through an alarm function $A$ in each of the considered magnitude ranges. For example, the alarm function of a rate-based model may be simply calculated as a sum of the rates over a magnitude range (Kossobokov 2006). 
In a Molchan diagram, we can define the probability gain as

$$
G_{\text {input }}^{\text {current }}\left(A_{0}\right)=\frac{(1-v)}{\tau}=\frac{\sum_{A_{0} \leq A(x, t)} \omega(x, t)}{\sum_{A_{0} \leq A(x, t)} \lambda(x, t)} \times \frac{\sum \lambda(x, t)}{\sum \omega(x, t)},
$$

where $A_{0}$ is the threshold value of the alarm function, and the sum symbols refer to the space-time regions in which the subscript condition is satisfied. This $G$ value is a factor that integrates the increase of the rate of the current model within the space-time region in which $A>A_{0}$ (Aki 1981; Molchan 1991; Zechar and Jordan 2008). To isolate smaller areas and specific behaviors associated with different ranges of the alarm function, we work with the differential probability gain function that can be defined as the derivative of a continuous Molchan trajectory,

$$
g_{\text {input }}^{\text {current }}=-\partial \nu / \partial \tau .
$$

With the small samples we have in practice, the Molchan trajectory is always a steplike function. We smooth this function using a limited number of segments to avoid overfitting the differential probability gain function (see Appendix 1). Finally, for each segment and the corresponding range $\left[A_{0} ; A_{0}+\delta A_{0}\right]$ of alarm function values, we have a differential probability gain of

$g_{\text {input }}^{\text {current }}=-\Delta \nu / \Delta \tau=\frac{\sum_{A_{0} \leq A(x, t)<A_{0}+\delta A_{0}} \omega(x, t)}{\sum_{A_{0} \leq A(x, t)<A_{0}+\delta A_{0}} \lambda(x, t)} \times \frac{\sum \lambda(x, t)}{\sum \omega(x, t)}$,

where the sum is taken over to the space-time regions in which the subscript condition is satisfied. Considering all segments, we can assign a specific $g_{\text {input }}^{\text {current }}$ value to any $A$ value of the input model. Having done that, we can produce space-time maps of the differential probability gain of the input model and combine them with the current rate-based model. Then, the next generation of the rate-based model is defined as

$$
\lambda_{\text {new }}=g_{\text {input }}^{\text {current }}(A(x, t)) \lambda_{\text {current }},
$$

that is, the initial rates of the current model increase or decrease according to the local $g_{\text {input }}^{\text {current }}$ value. The $g_{\text {input }}^{\text {current }}$ values are always estimated retrospectively over long times to be used in future applications.

This method of model combination is similar to convolving the current model with the input model. For this reason, in the following, we denote one iteration of this procedure by

new model $=($ current model $) *($ input model $)$.

\section{Results and discussion}

Combining Every Earthquake a Precursor According to Scale and Early Aftershocks Statistics forecast models in California

The Every Earthquake a Precursor According to Scale (EEPAS) and Early Aftershocks Statistics (EAST) models are forecast models installed at the CSEP Testing Center in California. They are of special interest for this study because, based upon the joint evaluation using Molchan tests outside the official CSEP testing process, it was found that they both yield statistically significant better forecasts than a Relative Intensity (RI) reference model, a timeindependent model that is commonly used as a reference model in Molchan tests (Kossobokov and Shebalin 2003; Helmstetter et al. 2006; Molchan and Keilis-Borok 2008; Zechar and Jordan 2008).

The EEPAS model (Rhoades and Evison 2004; 2007) is a medium-term forecast model based on the precursory scale increase phenomenon and associated predictive scaling relations (Evison and Rhoades 2004). In this model, every earthquake is a precursor according to scale, with the scale referring to larger earthquakes to follow in the medium to long term. Then, smaller earthquakes are 'witnesses' of the seismogenic process and not 'actors' as in the well-known branching model ETAS (Ogata 1989). Several versions of the EEPAS model have been used to generate forecasts of seismicity for the next 3 months. They have been tested at the California CSEP testing center since January 2008. Among the five versions of this forecast model, we chose the EEPAS-0F model (henceforth referred to as simply the EEPAS model) because it performs the best against the RI reference model.

The EAST model is an alarm-based earthquake forecast model that uses early aftershock statistics (Shebalin et al. 2011). This model is based on the hypothesis that the time delay before the onset of the power-law aftershock decay rate decreases as the level of stress, and the seismogenic potential increase (Narteau et al. 2002; 2005; 2008; Narteau et al. 2009). In contrast to the EEPAS model, the EAST model is not a model of seismicity rates. Instead, it possesses a nonprobabilistic alarm function that is dedicated to detecting places with a higher level of stress where an earthquake is more likely to occur. These zones are identified by relatively low values of the geometric mean of elapsed times between mainshocks and early aftershocks. The three-month class EAST model has been archived at the California CSEP center since July 2009.

To perform all the likelihood tests for CSEP testing centers, a rate-based version of the EAST model has been developed by Shebalin et al. (2012). Here, this new rate-based model called $\mathrm{EAST}_{\mathrm{R}}$ can be described as a combination of EAST (i.e., the input model) and the RI time-independent reference model (i.e., the current model). If the new rate-based model performs similarly to 
the initial alarm-based model, its forecast skill is likely to depend on the conversion method. This is also true for the combining method developed here, especially since it can be applied to two time-dependent models.

In all our tests, we consider two testing regions: the official CSEP California testing region and a subset, with the idea that the subset will reduce the problem of reduced earthquake detectability in the ocean and outside the USA (Shebalin et al. 2011). We also consider a retrospective period from January 1984 to June 2009 and a quasiprospective time period from July 2009 to December 2011. The official CSEP test started on 1 July 12009 , for the EAST model and earlier for the EEPAS model, and all

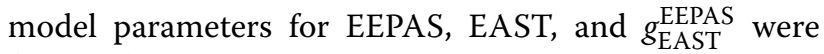
fixed beforehand. Note that to set up the EAST model and to calculate the functions $g_{\text {EAST }}^{\text {EEPAS }}$, we only use the testing region subset.

\section{Cross-evaluation of earthquake forecast models}

To underline how different forecast models may provide independent information about seismicity, we perform a cross-evaluation of the EAST $R$ and EEPAS models using Molchan diagrams (Figure 1). In both retrospective (Figure 1a) and quasi-prospective tests (Figure 1b,c), Molchan trajectories are below the diagonal, indicating that each model provides a gain in prediction with respect to the other (see Subsection 'Molchan tests'). Although at first glance these results might appear contradictory, we interpret this as an indication that the EAST $\mathrm{R}_{\mathrm{R}}$ and EEPAS models are complementary. Because they focus on different relevant aspects of seismicity, each of them gives an additional amount of predictive information.
This complementary nature of two independent models of seismicity is difficult to detect using likelihood tests (Table 1). However, we stress the point that it may be an important property for earthquake forecasting and certainly the best case to combine two independent models. Then, the method to combine must preserve the knowledge gain that each model offers.

Here, we use the differential probability gain method to combine two forecast models. We infer that the increase in expected rates may be locally high, particularly if several models are successively combined. For one iteration, the combination is driven by the slope of the Molchan trajectories. Therefore, it is ideal to have two models that are substantially complementary in their forecasts. As shown by Figure 1, this condition is satisfied by the EAST and EEPAS models, and their combination may yield new predictive information on California seismicity.

\section{A combination of EAST and EEPAS forecast models using differential probability gains}

As shown by (Shebalin et al. 2012), there is no significant difference in the predictive power of the EAST and EAST $T_{R}$ models. Therefore, to avoid potential noise introduced by the RI reference model or the conversion method, we combine directly the EAST and EEPAS models. To construct the differential probability gain functions $g_{\text {EAST }}^{\text {EEPAS }}$, we consider the retrospective period and three magnitude ranges of $[4.95 ; 5.45),[5.45 ; 5.95)$, and $[5.95 ; \infty)$. Within each of those intervals, the combined model inherits the magnitude distribution of the EEPAS model, which is not constrained to follow the Gutenberg-Richter relation in each cell. Figure 2 shows for each interval the Molchan
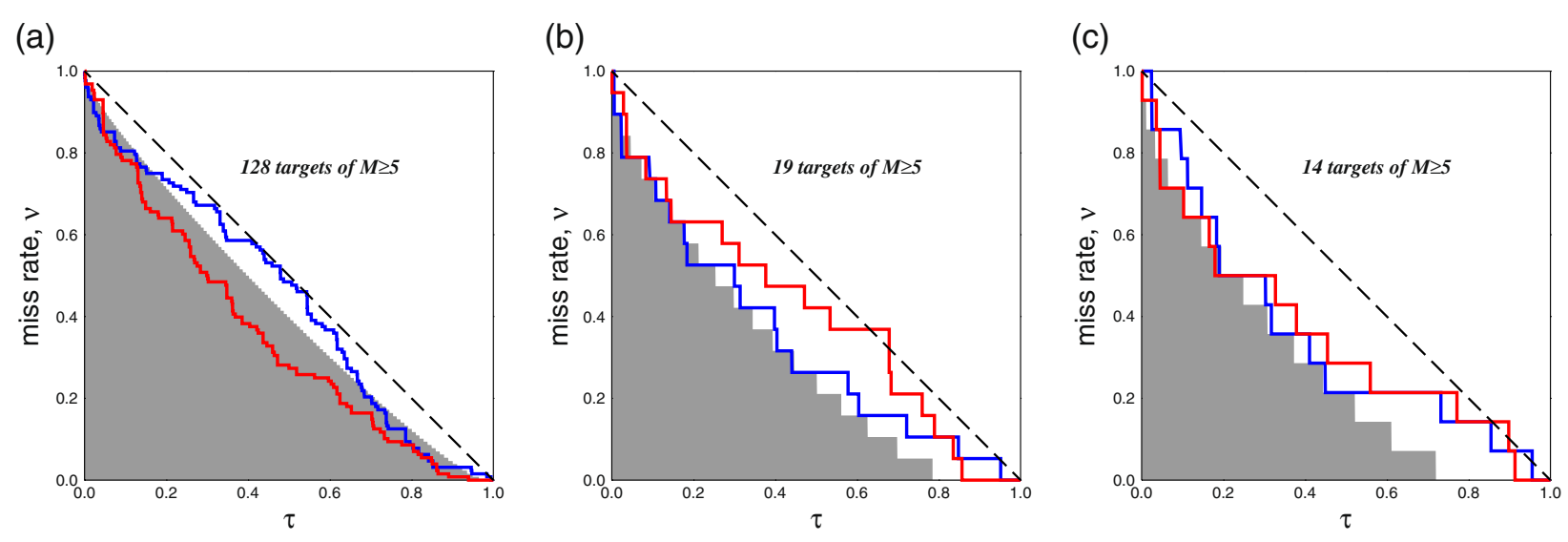

Figure 1 Cross-evaluation of the EEPAS and EAST $T_{\mathrm{R}}$ models in California for $M \geq 4.95$ target earthquakes. (a) Retrospective period from January 1984 to June 2009. (b and c) Quasi-prospective period from July 2009 to December 2011. We consider the entire CSEP testing region in (b) and a reduced region in (a) and (c) to exclude off-coast and outside USA areas (Shebalin et al. 2011). Using Molchan diagrams, we compare the forecasts of the EASTR model with respect to the EEPAS model (red lines) and vice versa (blue lines). The dashed diagonal line corresponds to an unskilled forecast. The shaded area indicates the zone in which the prediction of the tested model outperforms the prediction of the reference model at a level of significance $\alpha=1 \%$. For both the EEPAS and the EAST $T_{R}$ models, we consider single rate values obtained by summing the expected rates of $M \geq 4.95$ target earthquakes. 
Table 1 Complete and spatial likelihood results for the forecasts

\begin{tabular}{|c|c|c|c|c|c|c|}
\hline Period & $N_{\text {target }^{\mathrm{a}}}$ & EAST $*$ EEPAS & EAST + EEPAS & $\mathrm{EAST}_{R}$ & EEPAS & RI \\
\hline \multicolumn{7}{|l|}{ Complete likelihood } \\
\hline Jul-Aug 2009 & 2 & -13.62 & -15.71 & -15.96 & -15.58 & -18.64 \\
\hline Sep-Dec 2009 & 2 & -17.65 & -18.82 & -19.42 & -18.44 & -20.93 \\
\hline Jan-Mar 2010 & 2 & -24.97 & -24.56 & -26.76 & -23.57 & -25.17 \\
\hline Apr-Jun 2010 & 8 & -74.79 & -76.13 & -77.82 & -77.35 & -81.69 \\
\hline Jul-Aug 2010 & 2 & -14.87 & -14.25 & -17.47 & -16.71 & -19.65 \\
\hline Sep-Dec 2010 & 0 & -2.18 & -1.88 & -1.36 & -2.40 & -1.78 \\
\hline Jan-Mar 2011 & 1 & -8.22 & -7.94 & -11.62 & -7.70 & -11.07 \\
\hline Apr-Jun 2011 & 1 & -12.50 & -12.23 & -12.12 & -12.40 & -11.55 \\
\hline Jul-Aug 2011 & 0 & -1.75 & -1.74 & -1.36 & -2.12 & -1.78 \\
\hline Sep-Dec 2011 & 1 & -13.48 & -12.81 & -12.29 & -13.41 & -11.66 \\
\hline Jul 2009-Dec 2011 & 19 & -184.04 & -186.07 & -196.17 & -189.66 & -203.91 \\
\hline Jul 2009-Dec 2011 b & 14 & -137.68 & -140.08 & -138.63 & -146.48 & -151.46 \\
\hline \multicolumn{7}{|l|}{ Spatial likelihood } \\
\hline Jul-Aug 2009 & 2 & -10.64 & -12.45 & -12.67 & -12.46 & -15.15 \\
\hline Sep-Dec 2009 & 2 & -13.44 & -13.68 & -15.15 & -13.15 & -15.47 \\
\hline Jan-Mar 2010 & 2 & -16.18 & -16.27 & -17.76 & -15.40 & -16.65 \\
\hline Apr-Jun 2010 & 8 & -42.07 & -44.76 & -45.20 & -46.11 & -51.93 \\
\hline Jul-Aug 2010 & 2 & -11.49 & -10.69 & -13.75 & -12.81 & -15.51 \\
\hline Sep-Dec 2010 & 0 & 0.00 & 0.00 & 0.00 & 0.00 & 0.00 \\
\hline Jan-Mar 2011 & 1 & -6.24 & -6.01 & -9.84 & -5.55 & -9.02 \\
\hline Apr-Jun 2011 & 1 & -10.70 & -10.42 & -10.41 & -10.43 & -9.72 \\
\hline Jul-Aug 2011 & 0 & 0.00 & 0.00 & 0.00 & 0.00 & 0.00 \\
\hline Sep-Dec 2011 & 1 & -11.64 & -10.98 & -10.61 & -11.36 & -9.83 \\
\hline Jul 2009-Dec 2011 & 19 & -122.40 & -125.27 & -135.39 & -127.27 & -143.29 \\
\hline Jul 2009-Dec 2011 b & 14 & -88.93 & -92.39 & -92.45 & -96.62 & -103.87 \\
\hline
\end{tabular}

${ }^{a} N_{\text {target }}$ is the number of $M \geq 4.95$ earthquakes during the indicated periods.

${ }^{b}$ Reduced region to exclude off-coast and outside USA areas. Complete and spatial likelihood results for the forecasts of the EAST $* E E P A S$, linear combination EAST+EEPAS (half and half), EAST ${ }_{R}, E E P A S$, and RI reference models.

diagrams, their approximation by segments, and the differential probability gain functions of the EAST model with respect to the EEPAS model. We observe that the $g_{\text {EAST }}^{\text {EEPAS }}$ values are greater than one for almost two orders of magnitude of the alarm function of the EAST forecast model (Figure 2d,e,f).

For three magnitude intervals we use the corresponding functions $g_{\text {EAST }}^{\text {EEPAS }}$ and the rates $\lambda$ EEPAS of the EEPAS model in Equation 6 to obtain the rates of the new rate-based model EAST $*$ EEPAS. Figure 3 shows Molchan diagrams used to evaluate the forecast of the EAST, EEPAS, and EAST $*$ EEPAS models with respect to the RI reference model during the quasi-prospective period. The comparison of these Molchan trajectories shows that the combined model works better than the two initial models from which it has been derived. This is particularly the case for the smallest $\tau_{\mathrm{RI}}$ value, for which both initial models perform better than the RI reference model at a significance level below $\alpha=1 \%$. Results of total likelihood and spatial likelihood (Table 1) indicate also a gain of the EAST $*$ EEPAS model with respect to the EEPAS model. Quantitatively, the log-likelihood gain is 0.30 and 0.26 per earthquake for total and spatial likelihoods, respectively. With respect to the $\mathrm{EAST}_{\mathrm{R}}$ model, these gains are 0.34 and 0.43 , respectively.

During the quasi-prospective period, many of the target earthquakes occurred in the ocean or outside the USA. In those zones, the catalog of $M \geq 1.8$ early aftershocks is incomplete and the alarm function of the EAST model is less precisely defined (Shebalin et al. 2011). In the subset of California that we used in the retrospective analysis, the likelihood gain of the EAST $*$ EEPAS model with respect to the EEPAS model is more than 0.5 per earthquake. The gain with respect to the $\mathrm{EAST}_{\mathrm{R}}$ model 
(a)

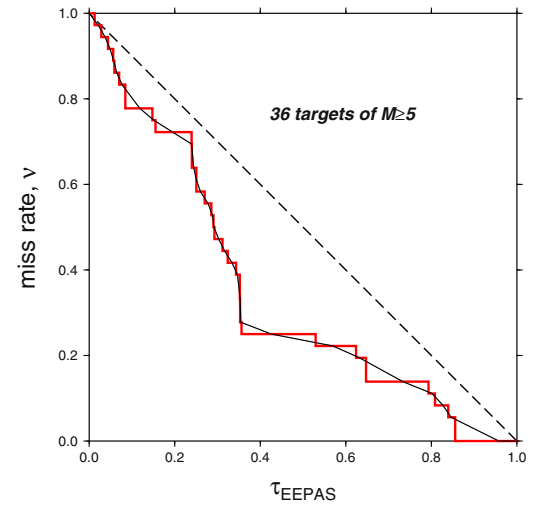

(d)

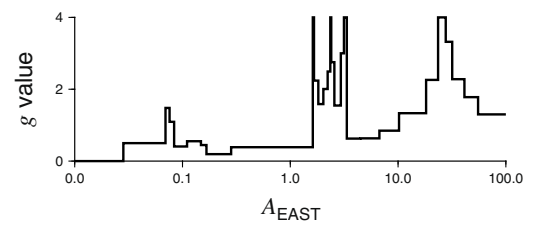

(b)

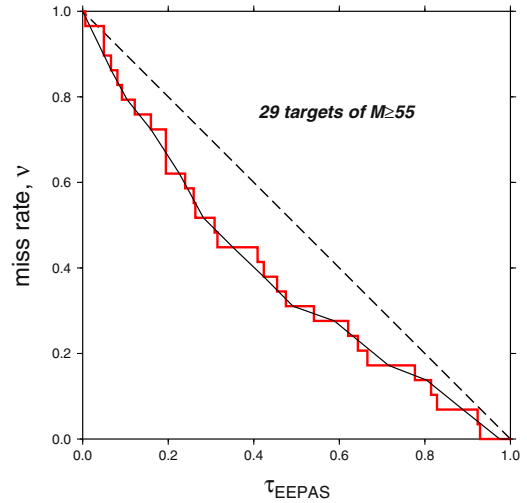

(e)

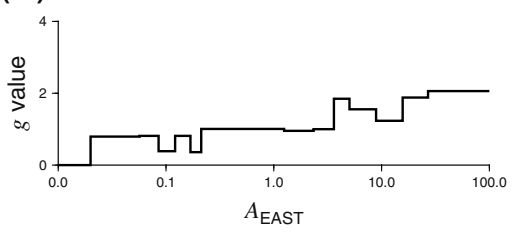

(c)

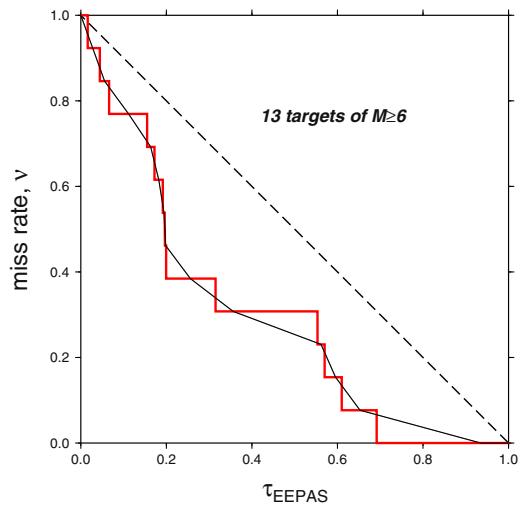

(f)

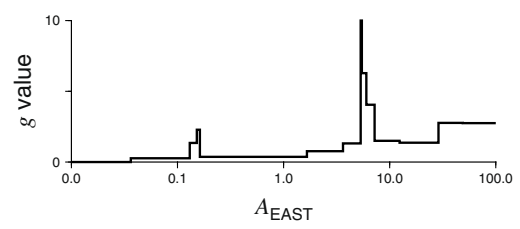

Figure 2 Differential probability gain functions for EAST model with respect to EEPAS model. Estimation of the differential probability gain functions, $g_{\text {EAST }}^{\text {EEPS }}$ of the EAST forecast model with respect to the EEPAS model for California from January 1984 to June 2009. (a, b, and c) Molchan diagrams, in which we smooth the Molchan trajectory (red line) by a set of segments (black lines; see Appendix 1). The $g_{\text {EAST }}^{\text {EEAS }}$ value is the local slope of these segments. (d, e, and f) Differential probability gain $g_{\text {EAST }}^{\text {EEAS }}$ as a function of the alarm function $A_{\text {EAST }}$ of the EAST models. We use three nonoverlapping magnitude intervals of $[4.95 ; 5.45)$ in $\mathbf{( a )}$ and $(\mathbf{d}),[5.45 ; 5.95)$ in (b) and (e), and $[5.95 ; \infty)$ in (c) and (f). Note that for (a) and (d), we use a shorter time period from January 1995 to June 2009 to eliminate aftershocks of the Landers earthquake.

(a)

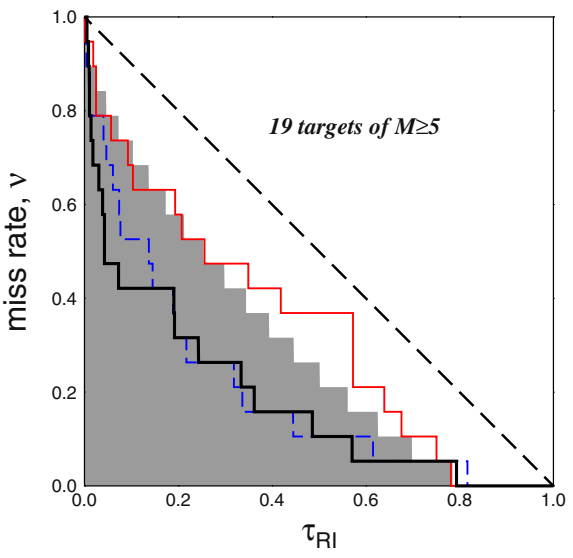

(b)

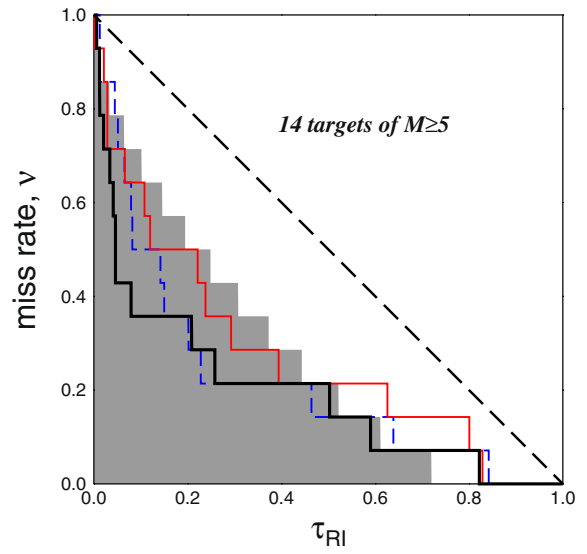

Figure 3 Quasi-prospective evaluation of the EAST $* E_{E P A S}$, EAST $_{\mathrm{R}}$, and EEPAS models. Quasi-prospective evaluation (July 2009 to December 2011) of the EAST*EEPAS, EAST $R$, and EEPAS models with respect to the RI reference model in California. Tests were done in the entire California CSEP testing region (a) and in a reduced region (b) that does not include off-coast and outside USA areas (Shebalin et al. 2011). Using Molchan diagrams, we compare the forecasts of the EAST*EEPAS (black lines), EAST $R$ (red lines), and EEPAS (blue dashed lines) models with respect to the RI reference model. The dashed diagonal line corresponds to an unskilled forecast. The shaded area indicates the zone in which the forecast of the tested model outperforms the forecast of the reference model at a level of significance $\alpha=1 \%$. For all the models, we consider single rate values obtained by summing the expected rates of $M \geq 4.95$ target earthquakes. 
is smaller, 0.05 for total likelihood and 0.25 for spatial likelihood.

Figure 4 illustrates how our combining method works using the outputs of the EAST, EAST $T_{R}, E E P A S$, and EAST $*$ EEPAS models for the forecast period from April to June 2010 along the USA-Mexico border. This spacetime region includes the $M 7.2$ El Mayor-Cucapah earthquake of 4 April 2010. Figure 4a shows the map of the EAST alarm function values. For rate-base models (Figure $4 \mathrm{~b}, \mathrm{c}, \mathrm{d}$ ), the rates are calculated for $M \geq 4.95$ events by summation of all model rates in corresponding magnitude bins. All models exhibit a clear maximum near the epicenter of the El Mayor-Cucapah earthquake. However, in an area extending northward from this epicenter, the EAST $*$ EEPAS model gives rates that are almost one order of magnitude larger than the rates of the two individual models. This example demonstrates that our combining method sharpens these individual forecasts, providing higher expected earthquake rates in more confined areas. These local increases of the forecast event rates are compensated by decreases in other places so that the total event rate over the whole territory does not significantly change (see Appendix 2).

\section{Comparing combining methods}

Using the weighted average of two rate-based models is straightforward and therefore the most common combining method (Rhoades and Gerstenberger 2009; Marzocchi et al. 2012) . In addition, the use of weighted averages may increase the total predictive skill of the resulting model by locally giving more importance to high and low extreme rate values of each model. However, it remains an averaging method. Then, if the combined model keeps the total expected earthquake rate (convex combination) unchanged, local rates cannot be higher (or lower) than the maximum (minimum) rates of the two models. One exception is the combination of models that concentrate on forecasting different seismic patterns (for example, so-called mainshocks and aftershocks (Rhoades and Gerstenberger 2009)). In that case, it is not necessary to keep the total rate unchanged, and the combined rates may exceed the maximum of the two models being combined.

Here, we compare the EAST $*$ EEPPAS model to the $E_{R A S T}+$ EEPAS model, the simple average of the EAST and EEPAS forecast models. Then, using Molchan diagrams, we compare the two combined models to the RI reference model. Figure 5 shows that the forecast of the EAST $*$ EEPAS model outperforms the forecast of the $E_{A S T}+E E P A S$ model, especially for the smallest $\tau_{\mathrm{RI}}$ value, for which both the EAST and EEPAS models perform the best.

The results of the likelihood tests are quite different than those for Molchan diagrams. In fact, if the Molchan trajectory of a linearly combined model is likely to run between the trajectories of the initial models, Table 1 shows that the log likelihood for the linearly combined model is closer to zero than for the models from which it is derived. However, the EAST $*$ EEPAS model again exhibits better performance than the EAST $\mathrm{R}+\mathrm{EEPAS}$ model. The gain in log likelihood per earthquake is 0.11 for total likelihood and 0.15 for spatial likelihood. In the reduced region, the EAST $*$ EEPPAS performs better than the linearly combined model, which now has a score between that of EEPAS and that of EAST $\mathrm{R}$.

Figure $6 \mathrm{a}$ shows the cumulative distribution functions of the rates predicted by the EAST $*$ EEPAS and $E_{A S T}+E E P A S$ models. This plot indicates that the EAST $*$ EEPAS model explores a much wider range of values than the $\mathrm{EAST}_{\mathrm{R}}+\mathrm{EEPAS}$ models. In addition, we observe that more than $50 \%$ of target earthquakes occur for only $2 \%$ of the highest rates and that, for these events, the rates of the EAST $*$ EEPAS model are about twice those of the EAST $T_{R}+E E P A S$ model. This difference indicates that the combination based on differential probability gain is currently better than a linear combination at increasing the predicted event rates in the limit of high rate values. Obviously, the opposite is true in the limit of low range value. Nevertheless, in this case, both models fail to predict the target earthquake and a possible gain in trying to combine them is worth discussing. Simultaneously, these results confirm that the only restriction for performing a combination is the need to use two complementary forecast models, i.e., two models that ensure a nontrivial Molchan diagram with respect to one another $\left(g_{\text {input }}^{\text {current }}>1\right.$ in Equation 6).

Figure $6 \mathrm{~b}, \mathrm{c}$ compares the single rate values of the EAST $* E E P A S$ and EAST $_{\mathrm{R}}+$ EEPAS models in space-time regions where a target earthquake occurred. In the limit of high rates, the rate values of the EAST $*$ EEPAS model are double those of the EAST $\mathrm{R}_{\mathrm{R}}+$ EEPAS model (Figure $6 \mathrm{~b}$ ). This demonstrates that a combination of forecast models based on differential probability gains can increase earthquake probabilities in rate-based forecast models.

\section{Conclusions}

There are different types of earthquake forecast models, all of which are related to specific sets of observations (e.g., catalogs of seismicity, fault maps, strain rates, and seismic precursors). As illustrated by the outputs of alarm-based and rate-based models, individual forecasts may fall into different classes of statistical models. In this specific context, it is still impossible to combine all types of forecast using ensemble classification methods. Hence, we proposed here a practical method to combine forecasts based on differential probability gains.

This method can be described as an operational device to identify and join independent sources of information 

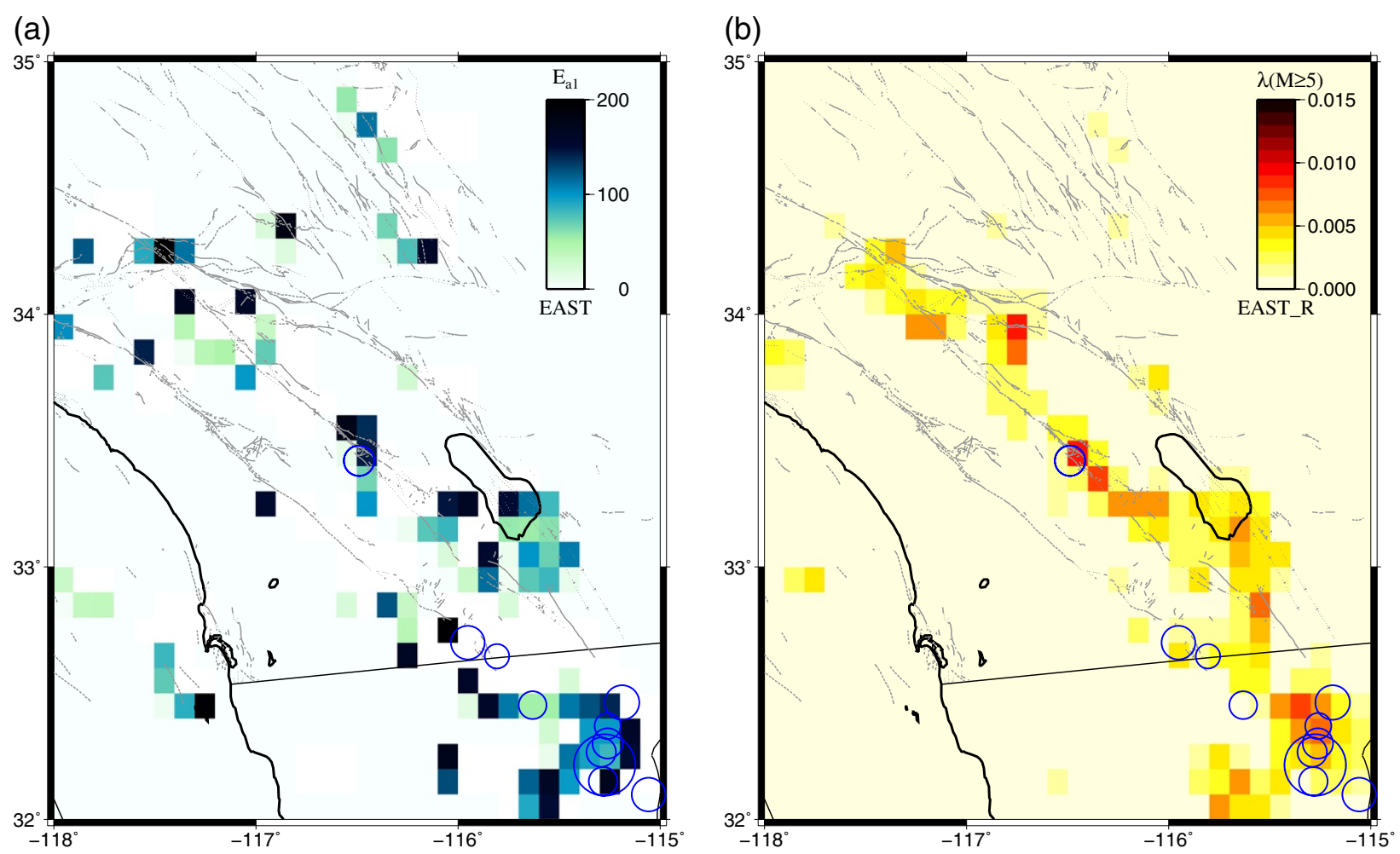

(c)

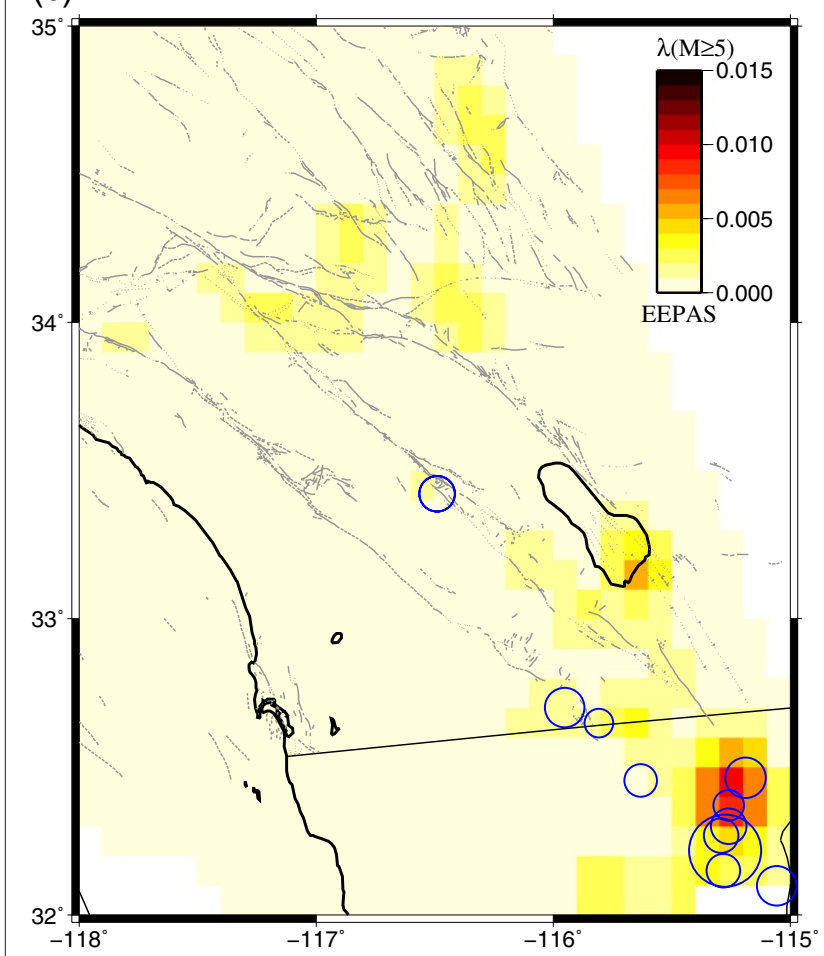

(d)

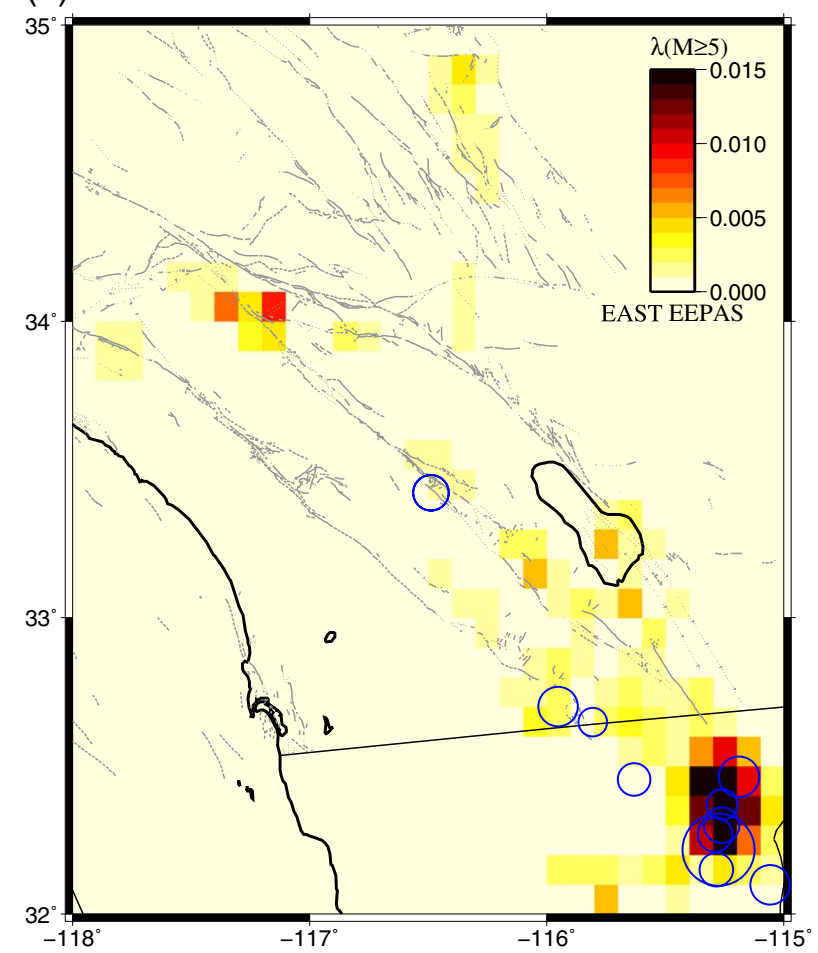

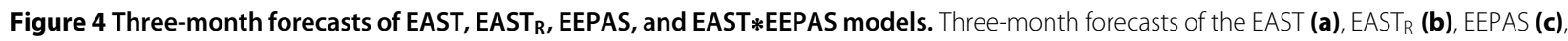
and EAST*EEPAS (d) models for $M \geq 4.95$ earthquakes from April to June 2010 in northern Baja, California along the USA-Mexico border. Blue circles correspond to $M \geq 4.95$ earthquakes that occurred in this area during this period. For the EAST model the color map varies from zero to the maximum of the alarm function $E_{a 1}$ (Shebalin et al. 2011). For other models, the same color bar is used to represent the forecast rates of $M \geq 4.95$

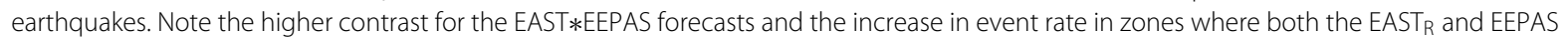
models have high event rates. Straight line is the USA-Mexico border. 
(a)

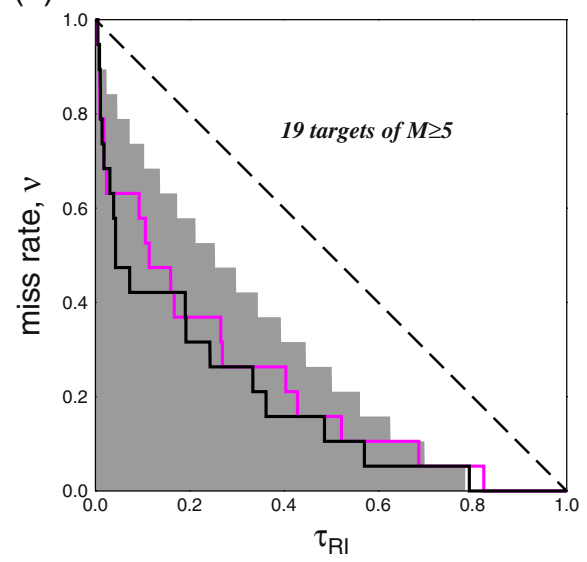

(b)

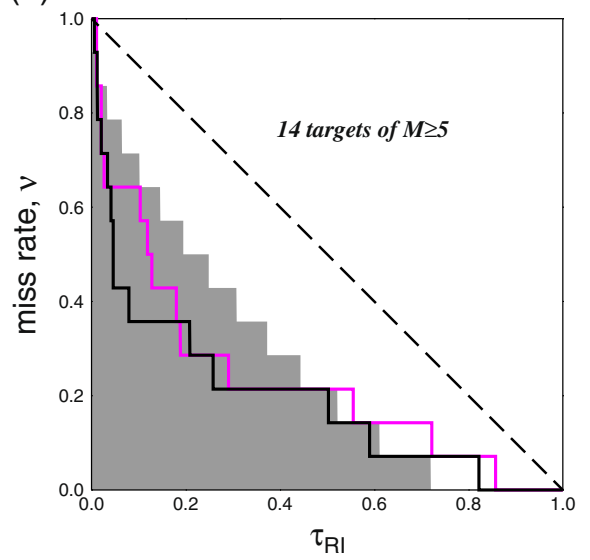

Figure 5 Comparison of forecasts of the EAST *EEPAS and the EAST + EEPAS models using Molchan diagrams. The tests were done in the entire California CSEP testing region (a) and in a reduced region (b) that does not include off-coast and outside USA areas (Shebalin et al. 2011). Using Molchan diagrams, we compare the forecasts of the EAST*EEPAS (black lines) and EAST + EEPAS (magenta lines) models with respect to the RI reference model. In the linear combination EAST $R+E E P A S$, the rates issued from both forecast models have the same weight. The dashed diagonal line corresponds to an unskilled forecast. The shaded area indicates the zone in which the forecast of the tested model outperforms the forecast of the reference model at a level of significance $\alpha=1 \%$. For both combined models, we consider single rate values obtained by summing the expected rates of $M \geq 4.95$ target earthquakes.

related to earthquake phenomena. The quality of the combined model does not have to depend on causal relationships between the different models being combined. Actually, the procedure applies to any forecast models that have additional forecast skill. Nevertheless, since we cannot formulate it in terms of traditional classification problems without a loss of generality, the overall performance of a combined forecast model can only be established on purely empirical grounds.
In contrast to linear methods, our procedure does not average the local expected rates of different models. Instead, it redistributes in space the rates of the current model according to the additional knowledge carried by the input model. Then, as shown by Figure 6, the combined model may cover a larger range of rate values, especially in the limit of high rates that are critical for operational forecast. An essential property of this redistribution process is to keep constant the total expected rate (a)

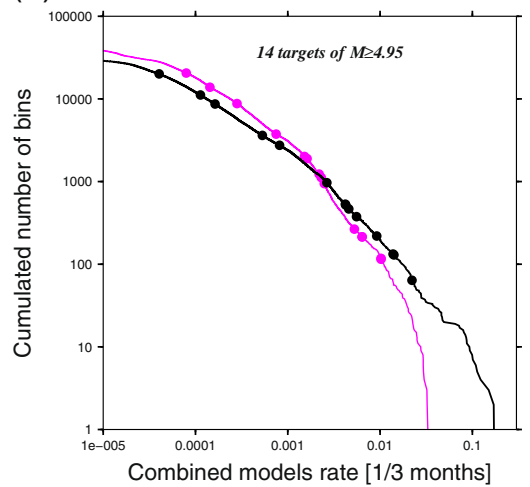

(b)

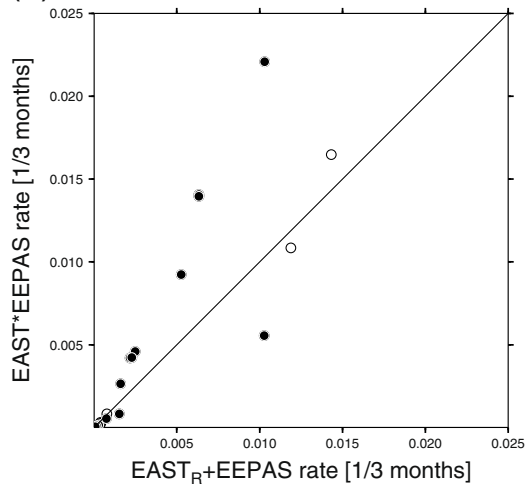

(c)

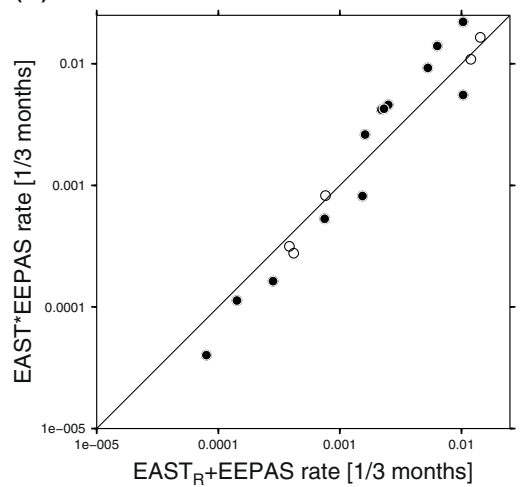

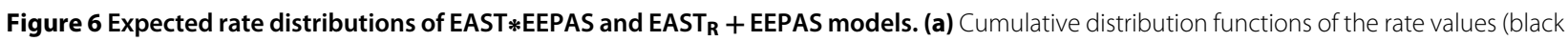
for EAST *EEPAS and magenta for EAST + EEPAS). Note the logarithmic scale. Dots show the rates in the space-time region where target earthquakes have occurred during the quasi-prospective test from July 2009 to December 2011. Linear-scale (b) and logarithmic-scale (c) rates of the EAST*EEPAS model with respect to rates of the EAST $R$ +EEPAS model in space-time regions where target earthquakes have occurred. Open circles correspond to the entire CSEP testing region; black dots correspond to the reduced region that does not include off-coast and outside USA areas (Shebalin et al. 2011). The $x=y$ line is shown for direct comparison. The curves in (a) are left-truncated at a rate of $10^{-5}$ per 3 months. The EAST $*$ EEPAS model has remarkably higher rate values in the limit of large rate (b). These higher rates are compensated in the model by lower rates in the limit of small rate. 
of the current rate-based model. This property can be easily demonstrated from simple geometric considerations on the smoothed Molchan trajectories (see Appendix 2 and Figure 2). Nevertheless, if this conservation property is verified for the retrospective period during which the differential probability functions have been determined, it may be only approximate during the quasi-prospective and real-time tests.

The differential probability gain approach can be used to combine successively different forecast models. However, each model brings not only additional information but also some noise. Therefore, changes in the level of noise must be estimated when many models are combined together. In real-time testing, it is practically impossible to quantify the level of noise in individual models because the available case histories are always quite short. Accordingly, making theoretical estimates of the overall noise is not yet possible. Instead, we perform numerical experiments (see Appendix 3). The test shows that even after 10 iterations with highly noisy simulated models, the result remains quite similar to the original one.

Our combination method is not commutative. To demonstrate this, we analyze the two combined models that can be derived from EAST $_{\mathrm{R}}$ and EEPAS models. We note that the two possible combined models are quite similar. Nevertheless, using Molchan and likelihood tests, we observe that the $\mathrm{EAST}_{\mathrm{R}} * \mathrm{EEPAS}$ model performs better (and is therefore different) than the EEPAS $* E A S T_{\mathrm{R}}$ model. The difference in log likelihood per earthquake is about 0.1 .

As shown in Figure 2d,e,f, we obtain nonmonotonic differential probability functions $g_{\text {EAST }}^{\text {EEPAS }}$, that is, the highest alarm function values do not always correspond to the highest $g_{\text {EAST }}^{\text {EEPAS }}$ values and high peaks may be observed (Figure 2d). Such cases require special attention. For example, it might be that the peaks are caused by aftershocks of a large earthquake. In our particular case, we checked the time and location of the earthquakes corresponding to the peaks and found only one case of spatial clustering, a swarm of four $4.97 \leq M \leq 5.1$ events in February 2008 preceded by a $M 5.36$ event in May 2006 and followed by a $M 4.96$ event in November 2008. This sequence took place near the epicenter of the future $M 7.2$ El Mayor-Cucapah earthquake of April 4, 2010. All these $M \approx 5$ events are associated with a multiple peak in Figure $2 \mathrm{~d}$ for $A_{\mathrm{EAST}} \approx 2$. However, this specific range of alarm function is also associated with 13 other target earthquakes. Hence, we infer that a monotonic character of the differential probability gain function $g$ is not a necessary condition to combine two models. The gain functions depend on the choice of the learning interval. Some stronger smoothing or approximation might be preferable, particularly for operational forecasts.
The major difference between Molchan and likelihood tests resides in the way the rate variable is weighted. Molchan tests are based on the sum of the expected event rates, whereas likelihood tests are based on the sum of the logarithm of these rates. The comparison of Figure 6b,c illustrates the difference between these two tests:

1. In Molchan diagrams, the relative performance of two models may be measured by the probability gain (see Equation 3, Aki (1981), and Molchan (1990)). For two rate-based models, this probability gain may also be estimated as the slope of the best-fit line in a cross-distribution of the rates (i.e., the diagram in which the rates of one model are plotted with respect to the other where target earthquakes have occurred). In Figure 6b, this slope is close to 2. In addition, we can graphically verify that events at high rates have more weight than events at small rates in determining this slope.

2. In likelihood tests, the relative performance of two models is expressed as the difference of their log likelihoods per earthquake (Equation 2). If the total expected rates are the same in both models, this is equivalent to the average vertical distance to the diagonal in Figure 6c. In contrast to Aki's probability gain (Figure 6b), this averaging gives the same weight to all rates. As a consequence, positive distances at high rates and negative distances at low rates cancel each other out.

This comparison highlights the advantage of our method based on Molchan tests. Indeed, good forecasts for low earthquake rates are not as important as for high rates. In fact, earthquakes occurring in space-time regions with low expected rates have to be considered as a 'failure to forecast'.

The combining method based on differential probability gains shows promising results compared to individual earthquake forecast models and other linear combination techniques. Overall, this procedure opens new opportunities for operational forecasting by substantially increasing the forecast event rates. One can also imagine applying an iterative application of our method to combine several forecast models of different types using the differential probability gain method. At each step, any model that does not provide additional knowledge with respect to the current model would be ignored in this combination. Therefore, the combination will be best if the input models are constructed from different concepts, data, or seismic precursors. A potential important research path would be to extend the scope of the current-generation earthquake forecast experiments to move beyond testing various models and begin evaluating different methods of model combination. 


\section{Appendix 1}

\section{Automatic procedure to estimate differential probability gain functions}

Given a finite number $N$ of target earthquakes and the discretization of space, the Molchan trajectory is a steplike function. To estimate the differential probability gain function, we use a procedure that automatically smooths

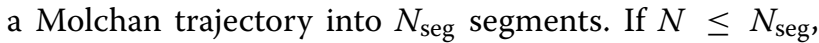
we consider a segment for each step of the Molchan trajectory, and the vertical coordinates of the segments are $i / N$ with $i \in\{0,1, \ldots, N\}$. If $N>N_{\text {seg, we only con- }}$

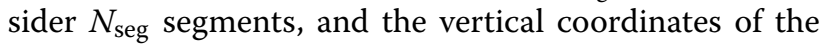
segments are $\left\lfloor N\left(N_{\text {seg }}-i\right) / N_{\text {seg }}\right\rfloor / N$ with $i \in[0, N]$ and where $\lfloor x\rfloor$ is the largest integer less than or equal to $x$. At each step where there is a vertical limit of segments, the horizontal coordinate of the segments is the $\tau$ value that corresponds to the median of the distribution of the alarm function value for this step. Everywhere in this study, we use $N_{\text {seg }}=20$ (Figure 2), but we check the stability of the results for $10<N_{\text {seg }}<30$. From our experience, we also infer that there is not a strong impact of the smoothing method on the result as soon as $N_{\text {seg }}>10$.

\section{Appendix 2}

\section{Conservation of the total expected rate in a combining} method using differential probability gains

$g(A)$ is the differential probability gain function of the Molchan diagram that evaluates the performance on an input model of alarm function $A$ with respect to a ratebased forecast model. $\lambda_{\text {current }}(x, t)$ are the expected event rates of this current version of the rate-based model in the space-time region $(x, t)$. Using our combining method, we calculate the expected event rates of the new rate-based model

$$
\lambda_{\text {new }}(x, t)=g(A(x, t)) \lambda_{\text {current }}(x, t) .
$$

To estimate the differential probability gain function, we smooth the Molchan trajectory using $N_{\text {seg }}$ segments (see Appendix 1). Let us denote by $\left(\tau_{i}, v_{i}\right)$ and $A_{i}, i \in$ $\left\{0,1, \ldots, N_{\text {seg }}\right\}$, the segment extremity coordinates and the corresponding alarm function values of the input model, respectively. The slope of these segments is the differential probability gain $g_{i}, i \in\left\{1,2, \ldots, N_{\text {seg }}\right\}$. By definition, we have

$$
g_{i}=\frac{\nu_{i}-v_{i-1}}{\tau_{i}-\tau_{i-1}} .
$$

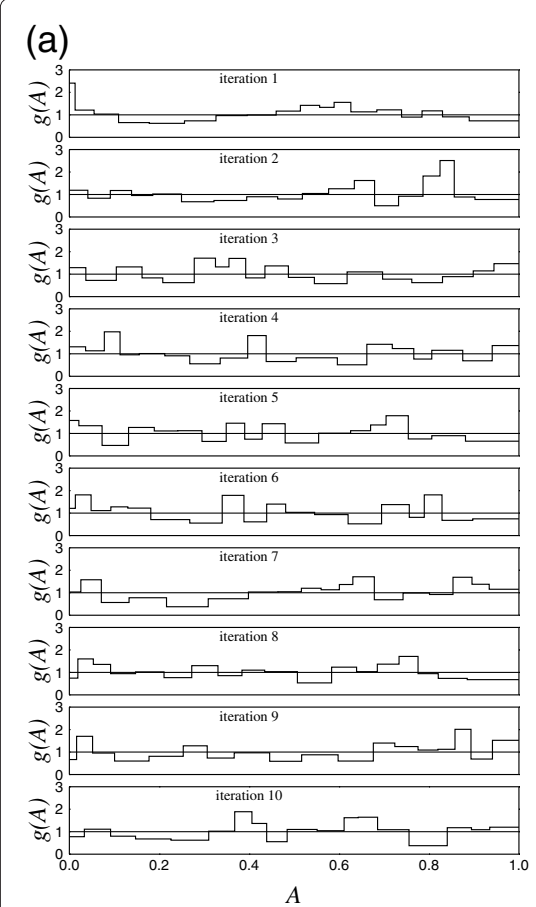

(b)

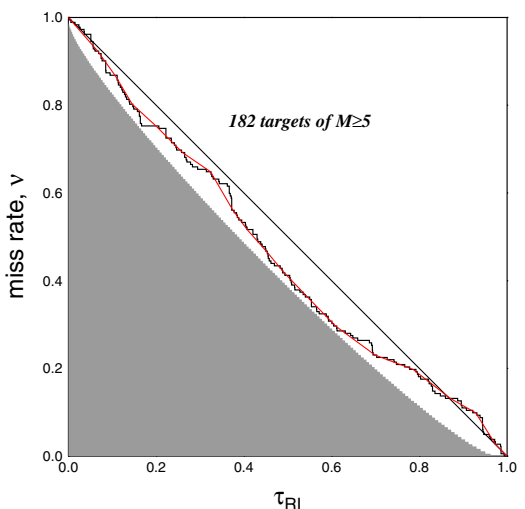

(c)

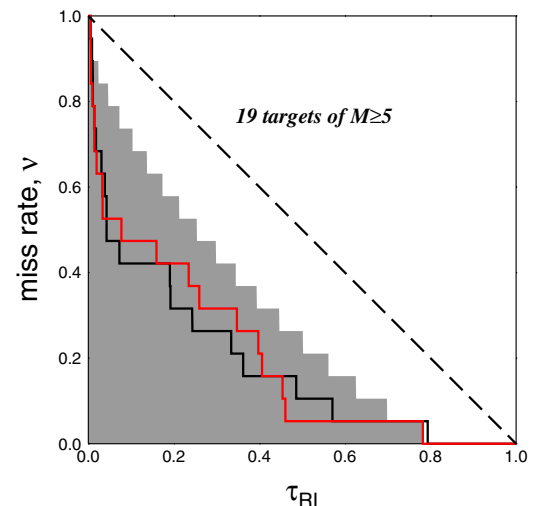

Figure $\mathbf{7}$ Level of noise in combined forecast models using the differential probability gains approach. The EAST $* E E P A S$ model is successively combined with 10 random rate-based models for $M>4.95$ target earthquakes (see text). (a) Differential probability gain $g(A)$ estimated during the learning period from January 1984 to June 2009. (b) For the same period, comparison of two consecutive generation forecasts before and after the 7th iteration using a Molchan diagram. This iteration exhibits the largest deviation from the diagonal. (c) Evaluation of the initial (black line) and final (red line) generation forecasts with respect to the RI reference model for the testing period from July 2009 to December 2011. In these Molchan diagrams, the dashed diagonal line corresponds to an unskilled forecast. The shaded area indicates the zone in which the forecast of the tested model outperforms the forecast of the reference model at a level of significance $\alpha=1 \%$. 
For the current and the new model, we may group the expected event rates according to the ranges of the input model alarm function that correspond to the different segments of the Molchan diagram (Figure 2 and Appendix 1):

$$
\begin{aligned}
\lambda_{\text {current }}^{i} & =\sum_{A_{i-1}<A(x, t)<A_{i}} \lambda_{\text {current }}(x, t), \\
\lambda_{\text {new }}^{i} & =\sum_{A_{i-1}<A(x, t)<A_{i}} \lambda_{\text {new }}(x, t),
\end{aligned}
$$

where the sum symbols refer to the space-time regions in which the subscript condition is satisfied. We define the total rates of the current and the new models as

$$
\Lambda_{\text {current }}=\sum_{i=1}^{N_{\text {seg }}} \lambda_{\text {current }}^{i} \quad \text { and } \quad \Lambda_{\text {new }}=\sum_{i=1}^{N_{\text {seg }}} \lambda_{\text {new }}^{i}
$$

For the period in which the differential probability gain function is estimated, we have

$$
\lambda_{\text {current }}^{i}=\Lambda_{\text {current }}\left(\tau_{i}-\tau_{i-1}\right) .
$$

In that case, using successively Equations 7, 11, and 8, we obtain

$$
\begin{aligned}
\Lambda_{\text {new }} & =\sum_{i=1}^{N_{\text {seg }}} g_{i} \lambda_{\text {current }}^{i}=\Lambda_{\text {current }} \sum_{i=1}^{N_{\text {seg }}} g_{i}\left(\tau_{i}-\tau_{i-1}\right) \\
& =\Lambda_{\text {current }} \sum_{i=1}^{N_{\text {seg }}}\left(v_{i}-v_{i-1}\right)=\Lambda_{\text {current }} .
\end{aligned}
$$

For a real-time test or a quasi-prospective test, this conservation of the total expected rate is approximate.

\section{Appendix 3}

The level of noise in combined forecast models

Taking the EAST $*$ EEPAS model as the initial forecast model, we study the impact of highly noisy alarm-based models on the next-generation forecasts (Figure 3a). As before, we used the period from January 1984 to June 2009 for learning and the period from July 2009 to December 2011 for testing. For both periods, a single magnitude interval for target events $(M \geq 4.95)$, and each space-time region, we simulate 10 alarm-based models by drawing random numbers from a uniform distribution between 0 and 1 . Then, we iteratively create the nextgeneration forecasts by incorporating the predictive skills of individual models into the current-generation forecast. As described in Section 'A differential probability gain approach for combining two earthquake forecast models' and Appendix 1, the $g(A(x, t))$ values are estimated during the learning period (Figure $7 \mathrm{a}$ ) and then injected into the current-generation forecasts for both periods. For the learning period, Figure $7 \mathrm{~b}$ shows the Molchan diagram that compares the forecasts before and after the 7th iteration. This iteration exhibits the largest deviation from the diagonal. For the testing period, Figure 7c shows the Molchan diagram that evaluates the predictive skills of the initial and final forecast models with respect to the RI reference model. We find that even after 10 iterations with highly noisy simulated models, the result remains quite similar to the original one. This result is consistent with all the successive Molchan diagrams, which systematically show that two consecutive forecasts have no specific skills with respect to one another. This analysis reveals that, despite an unavoidable increase of noise, there is no systematic erosion of the forecast skills of a model during the combining procedure. Therefore, an obvious recommendation is to avoid combining weak models.

\section{Competing interests}

The authors declare that they have no competing interests.

\section{Authors' contributions}

PS conceived, designed, and coordinated the study, conducted the numerical tests, and wrote the manuscript. CN designed the study and wrote the manuscript. JDZ designed the statistical tests and wrote the manuscript. MH designed the statistical tests. All authors read and approved the final manuscript.

\section{Acknowledgements}

We are grateful to the editor and the reviewers for their valuable comments. This work was partially supported by the Russian Foundation for Basic Researches, Project No. 14-05-00541. The research was partially performed within the framework of the REAKT Project (Strategies and tools for Real-Time EArthquake Risk ReducTion) founded by the European Community via the Seventh Framework Program for Research (FP7), Contract No. 282862.

\section{Author details}

${ }^{1}$ Institute of Earthquake Prediction Theory and Mathematical Geophysics, 84/32 Profsouznaya, Moscow 117997 Russia. ${ }^{2}$ Equipe de Dynamique des Fluides Géologiques, Institut de Physique du Globe de Paris, Sorbonne Paris Cité, Univ Paris Diderot, UMR 7154 CNRS, 1 rue Jussieu, Paris 75238, France. ${ }^{3}$ Swiss Seismological Service, ETH Zurich, NO H 3, Sonneggstrasse 5, Zürich 8092, Switzerland. ${ }^{4}$ Institutes of Applied and Industrial Mathematics, Universtität Potsdam, POB 601553, Potsdam 14115, Germany.

Received: 4 December 2013 Accepted: 17 April 2014

Published: 23 May 2014

\section{References}

Aki K (1981) A probabilistic synthesis of precursory phenomena. In: Simpson DV, Richards PG (eds) Earthquake prediction. An international Review Manrice Ewing series. American Geophysical Union, Washington, DC, pp 566-574

Aki K (1996) Scale dependence in earthquake phenomena and its relevance to earthquake prediction. Proc Natl Acad Sci USA 93:3740-3747

Eberhard DA, Zechar JD, Wiemer S (2012) A prospective earthquake forecast experiment in the western Pacific. Geophys J Int 190(3):1579-1592

Evison FF, Rhoades DA (2004) Demarcation and scaling of long-term seismogenesis. Pure Appl Geophys 161:21-45. doi:10.1007/s00024-003-2435-8

Gerstenberger MC, Jones LM, Wiemer S (2007) Short-term aftershock probabilities: case studies in California. Seism Res Lett 78:66-77

Hastie T, Tibshirani R, Friedman J (2008) The elements of statistical learning, 2nd edn. Springer (Springer Series in Statistics), New York, USA

Helmstetter A, Kagan YY, Jackson DD (2006) Comparison of short-term and time-independent earthquake forecast models for southern California. Bull Seismol Soc Am 96:90-106

Jordan TH (2006) Earthquake predictability, brick by brick. Seismol Res Lett 
Jordan TH, Jones LM (2010) Operational earthquake forecasting: some thoughts on why and how. Seism Res Lett 81:571-574

Keilis-Borok V, Kossobokov V (1990) Premonitory activation of earthquake flow - algorithm M8. Phys Earth Planet Inter 61:73-83

Keilis-Borok V, Rotwain I (1990) Diagnosis of time of increased probability of strong earthquakes in different regions of the world - algorithm CN. Phys Earth Planet Inter 61:57-72

Kossobokov V (2006) Testing earthquake prediction methods: the west Pacific short-term forecast of earthquakes with magnitude $M_{w} \geq 5.8$. Tectonophysics 413:25-31

Kossobokov V, Shebalin P (2003) Earthquake prediction. In: Keilis-Borok VI, Soloviev AA (eds) Nonlinear dynamics of the lithosphere and earthquake prediction. Springer, Berlin-Heidelberg, pp 141-205

Marzocchi W, Zechar JD, Jordan TH (2012) Bayesian forecast evaluation and ensemble earthquake forecasting. Bull Seismol Soc Am 102:2574-2584. doi:10.1785/0120110327

Molchan G (1990) Strategies in strong earthquake prediction. Phys Earth Planet Inter 61:84-98

Molchan G (1991) Structure of optimal strategies in earthquake prediction. Tectonophysics 193:267-276

Molchan G (2010) Space-time earthquake prediction: the error diagrams. Pure Appl Geophys 167(8-9):907-917. doi:10.1007/s00024-010-0087-z

Molchan G (2012) On the testing of seismicity models. Acta Geophysica 60(3):624-637. doi:10.2478/s11600-011-0042-0

Molchan G, Keilis-Borok V (2008) Earthquake prediction: probabilistic aspect. Geophys J Int 173:1012-1017

Nanjo KZ, Tsuruoka H, Hirata N, Jordan TH (2011) Overview of the first earthquake forecast testing experiment in Japan. Earth Planets Space 63(3): 159-169

Narteau C, Shebalin P, Holschneider M (2002) Temporal limits of the power law aftershock decay rate. J Geophys Res 107. doi:10.1029/2002JB001868

Narteau C, Shebalin P, Holschneider M (2005) Onset of power law aftershock decay rates in Southern California. Geophys Res Lett 32 doi:10.1029/2005GL023951

Narteau C, Shebalin P, Holschneider M (2008) Loading rates in California inferred from aftershocks. Nonlin Proc Geophys 15:245-263

Narteau C, Byrdina S, Shebalin P, Schorlemmer D (2009) Common dependence on stress for the two fundamental laws of statistical seismology. Nature 462:642-645. doi:10.1038/nature08553

Ogata Y (1989) Statistical models for standard seismicity and detection of anomalies by residual analysis. Tectonophysics 169:159-174

Peresan A, Costa G, Panza G (1999) Seismotectonic model and CN earthquake prediction in Italy. Pure Appl Geophys 154:281-306

Rhoades DA (2013) Mixture models for improved earthquake forecasting with short-to-medium time horizons. Bull Seimol Soc Am 103:2203-2215. doi:10.1785/0120120233

Rhoades DA, Evison FF (2004) Long-range earthquake forecasting with every earthquake a precursor according to scale. Pure Appl Geophys 161:47-72. doi:10.1007/s00024-003-2434-9

Rhoades DA, Evison FF (2007) Application of the EEPAS model to forecasting earthquakes of moderate magnitude in southern California. Seismol Res Lett 78:110-115. doi:10.1785/gssrl.78.1.110

Rhoades DA, Gerstenberger MC (2009) Mixture models for improved short-term earthquake forecasting. Bull Seism Soc Am 99:636-646. doi:10.1785/0120080063

Romashkova LL, Kossobokov VG (2004) Intermediate-term earthquake prediction based on spatially stable clusters of alarms. Dokl Earth Sci 398:947-949

Schorlemmer D, Gerstenberger M, Wiemer S, Jackson DD, Rhoades DA (2007) Earthquake likelihood model testing. Seismol Res Lett 78:17-29

Shebalin P, Kellis-Borok V, Gabrielov A, Zaliapin I, Turcotte D (2006) Short-term earthquake prediction by reverse analysis of lithosphere dynamics. Tectonophysics 413:63-75

Shebalin P, Narteau C, Holschneider M, Schorlemmer D (2011) Short-term earthquake forecasting using early aftershock statistics. Bull Seimol Soc Am 101: 297-312. doi:10.1785/0120100119

Shebalin P, Narteau C, Holschneider M (2012) From alarm-based to rate-based earthquake forecast models. Bull Seimol Soc Am 102 doi:10.1785/0120110126
Sobolev GA, Chelidze TL, Zavyalov AD, Slavina LB, Nikoladze VE (1991) Maps of expected earthquakes based on a combination of parameters. Tectonophysics 193:255-265. doi:10.1016/0040-1951(91)90335-P

Sobolev GA, Tyupkin YS, Smirnov VB (1996) Method of intermediate term earthquake prediction. Doklady Akademii Nauk 347:405-407

Taroni M, Zechar J, Marzocchi W (2014) Assessing annual global M6+ seismicity forecasts. Geophys J Int 196(1):422-431

Tsuruoka H, Hirata N, Schorlemmer D, Euchner F, Nanjo KZ, Jordan TH (2012) CSEP testing center and the first results of the earthquake forecast testing experiment in Japan. Earth Planets Space 64(8):661-671

Zechar JD (2010) Evaluating earthquake predictions and earthquake forecasts: a guide for students and new researchers. Community Online Resource for Statistical Seismicity Analysis. pp 1-26. doi:10.5078/corssa-77337879

Zechar JD, Jordan TH (2008) Testing alarm-based earthquake predictions. Geophys J Int 172:715-724. doi:10.1111/j.1365-246X.2007.03676.x

Zechar JD, Jordan TH (2010) The area skill score statistic for evaluating earthquake predictability experiments. Pure Appl Geophys 167:893-906

Zechar JD, Gerstenberger MC, Rhoades DA (2010a) Likelihood-based tests for evaluating space--rate-magnitude earthquake forecasts. Bull Seism Soc Am 100(3):1184-1195. doi:10.1785/0120090192

Zechar JD, Schorlemmer D, Liukis M, Yu J, Euchner F, Maechling PJ, Jordan TH (2010b) The collaboratory for the study of earthquake predictability perspective on computational earthquake science. Concurrency Comput-Pract Exp 22:1836-1847

doi:10.1186/1880-5981-66-37

Cite this article as: Shebalin et al:: Combining earthquake forecasts using differential probability gains. Earth, Planets and Space 2014 66:37.

\section{Submit your manuscript to a SpringerOpen ${ }^{\circ}$ journal and benefit from:}

- Convenient online submission

- Rigorous peer review

- Immediate publication on acceptance

- Open access: articles freely available online

- High visibility within the field

- Retaining the copyright to your article

Submit your next manuscript at springeropen.com 